\title{
Peering into Past: What Happened to the Moon 3.6 Billion Years Ago?
}

\author{
Diamar Pechersky¹, Lev Eppelbaum² \\ ${ }^{1}$ Schmidt Institute of Earth Physics, Russian Academy of Sciences, Moscow, Russia \\ ${ }^{2}$ Department of Geosciences, Faculty of Exact Sciences, Tel Aviv University, Tel Aviv, Israel \\ Email: diamarmp@gmail.com, levap@post.tau.ac.il
}

How to cite this paper: Pechersky, D. and Eppelbaum, L. (2018) Peering into Past: What Happened to the Moon 3.6 Billion Years Ago? Positioning, 9, 73-78. https://doi.org/10.4236/pos.2018.93005

Received: July 17, 2018

Accepted: August 27, 2018

Published: August 30, 2018

Copyright (c) 2018 by authors and Scientific Research Publishing Inc. This work is licensed under the Creative Commons Attribution International License (CC BY 4.0).

http://creativecommons.org/licenses/by/4.0/

\begin{abstract}
Invaluable data can at times be overlooked or not fully exploited when first collected. Striking conclusions can often be drawn on the basis of a specific analysis many years later. The Apollo $11-17$ missions (1961-1972) provided detailed information on lunar basalts which make it possible to measure the iron grains in basalts from microphotographs of thin sections. Analysis of the average size of these grains $(D)$ differed as a function of the age of these basalts dated between 3.9 and 3.4 billion years ago, revealed that $D$ increased 1.5 fold, therefore the gravity acceleration $g$ decreased 1.5 fold. The intriguing conclusion can only be that the size of the Moon increased, its mass decreased, or both these factors have changed.
\end{abstract}

\section{Keywords}

Apollo 11 - 17 Missions, Lunar Basalts, "Iron Particles”, Moon Parameters

\section{Introduction}

Iron has always played a crucial role in geological evolution of the Earth and other cosmic bodies, although its value as a marker of key processes is often underestimated [1]. As shown elsewhere [2] [3], the vast majority of iron particles in sediments is of extraterrestrial origin, and is mainly composed of interplanetary space dust. However, these particles settle in sediments under influence of the Earth's gravity field. The average size of these iron particles, $D$, whether they are found in modern sediments and lavas, or in the Jurassic sediments of the Caucasus and Crimea and the Permian-Triassic traps of Siberia, is identical $(D \approx$ $21 \mu \mathrm{m})$.

Let us discuss iron particles inside the basalt-one of the most magnetized geological rocks [1]. These particles originated from the basaltic magma [1] [2] 
[3], apparently before its outpouring, and are under the constant influence of the gravitational field. For equal values of $D$, the force of gravity at the Earth's surface is considered to have been constant, at least in the Mesozoic and Cenozoic. One novel way to evaluate possible changes in the size of $D$ is to evaluate objects with a markedly different gravitational field; namely, lunar basalts.

The gravitational acceleration, $g$, on the Moon is six times less than on Earth (on Earth $g=9.807 \mathrm{~m} / \mathrm{s}^{2}$, on Moon $g=1.62 \mathrm{~m} / \mathrm{s}^{2}$ ). The Apollo $11-17$ [4] mission report provides a brief description of each basalt sample and photographs of thin sections; these data are of greatest importance [5]. In photos in reflected light, ore grains ranging from gray to bright white are visible, suggesting that the latter is most likely iron. In transmitted light we see only the black contours of all the ore minerals can be observed. In this case, we choose in photos the particles close to isometric and non-acute angled grains ranging in size from 4 - 5 to 100 - 120 microns. Not only iron, but also ilmenite, chromite, troilite and other ore minerals can mingle with these grains. We thus use the term "iron" in quotes to acknowledge this source of mixture, which can in fact be disregarded since all these minerals are noticeably heavier than basalt lava (the density of iron is 7900 $\mathrm{kg} / \mathrm{m}^{3}$, chromite is $4500-5000 \mathrm{~kg} / \mathrm{m}^{3}$, ilmenite is $4700 \mathrm{~g} / \mathrm{m}^{3}$, troilite is 4600 $\mathrm{kg} / \mathrm{m}^{3}$, basaltic lava is $2600 \mathrm{~kg} / \mathrm{m}^{3}$ ). Secondary changes of the "iron" grains are also irrelevant since we are only confident enough to see the contours of the grain. Locations of sampling areas on the Moon are shown in Figure 1 [6]. As

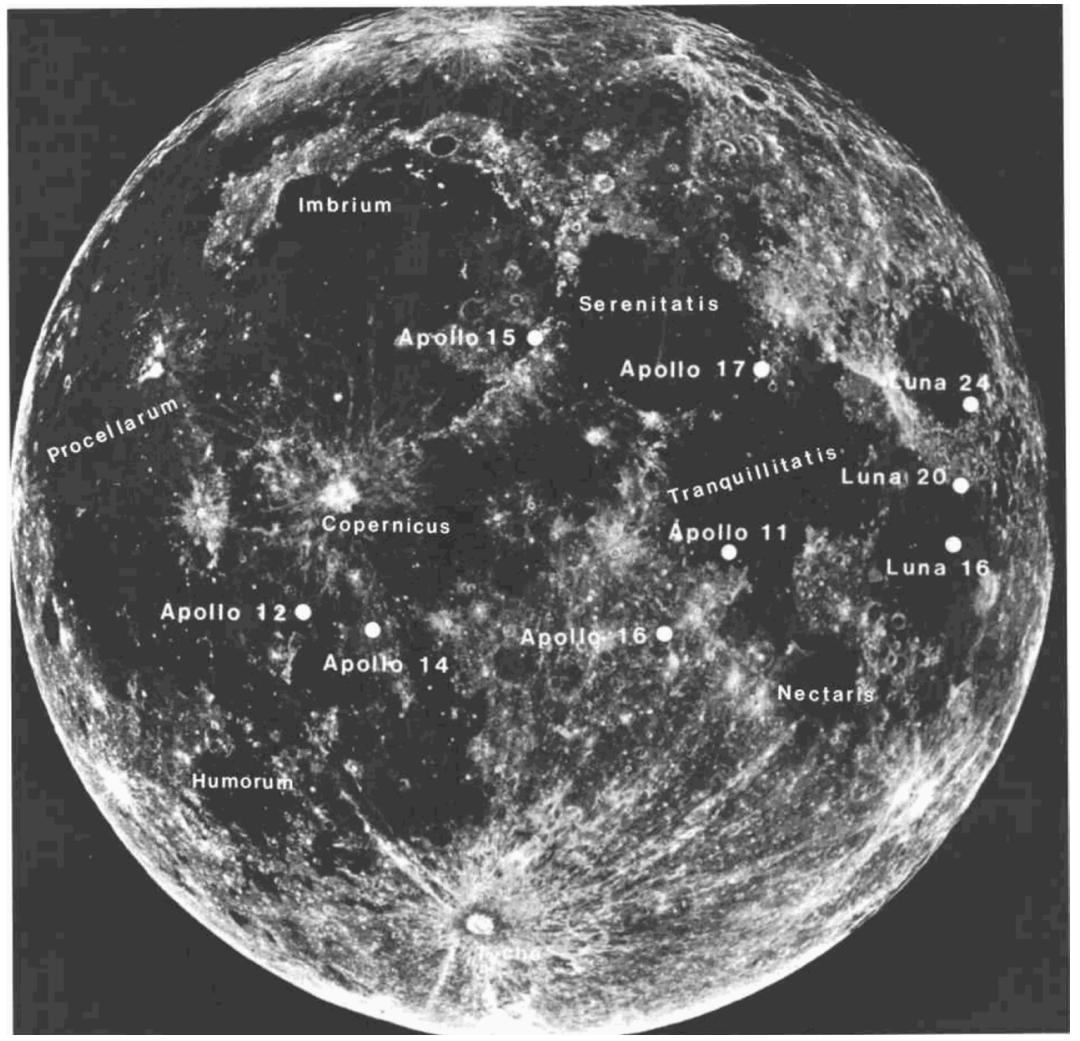

Figure 1. Location of Apollo 11 - 17 landing sites where rock samples were selected (on the basis of NASA Photo 584-31673) [6]. 
can be seen in the figure, the sampling sites are considerably removed from each other and cover a significant part of the lunar surface.

We predicted that when assuming equivalent conditions, with increasing $g$, the coarse grains should settle in the lava faster, at some intermediate level of the flow, such that the larger the $g$, the smaller the $D$ and vice versa-the smaller the $g$, the greater the $D$. Only samples with a known radiological age, and photographs of the sections in reflected (preferred) and transmitted light were included. As far as possible, we took the description [4] into account and excluded the edges of the flow (the presence of a firing zone, a preponderance of glass).

\section{Results of Measurements}

Table 1 and Figure 2 present the average sizes of the "iron" particles. The samples were examined and the grains of "iron" were analyzed much larger than those given in Table 1, because the table includes only those samples for which the radiological age appeared in the report [4].

Despite a wide spread of results, more noticeable for data in transmitted light, we found an explicit dependence of the average size $D$ of the "iron" grains on the age of the basalts, and consequently on the gravity field; specifically, from 3.9 to 3.4 billion years ago $(\mathrm{Ba})$ the field increased 1.5 fold. This can only mean that gravity decreased during the same period 1.5 fold, the Moon increased in size, or

Table 1. Average size of "iron" particles $D$ and age of lunar basalts (table is composed on the basis of report [4] examination).

\begin{tabular}{|c|c|c|c|c|c|c|c|}
\hline $\mathrm{N} / \mathrm{n}$ & Sample No. & $\begin{array}{l}\text { Apollo } \\
\text { Voyage }\end{array}$ & Petrographic type & $\begin{array}{c}\text { Regime of } \\
\text { mineralogical analysis }\end{array}$ & $N$ & $D$ & Age \\
\hline 1 & 10,003 & A-11 & medium-grained ilmenite pyroxene basalt & transm. light & 136 & 40.6 & 3.91 \\
\hline 2 & 10,017 & A-11 & fine-grained ilmenite pyroxene vesicular basalt & reflected light & 26 & 37.5 & 3.58 \\
\hline 3 & 10,045 & A-11 & fine-grained ilmenite olivine-pyroxene vesicular basalt & reflected light & 79 & 38.9 & 3.75 \\
\hline 4 & 12,022 & A-12 & medium-grained ilmenite-pyroxene basalt & reflected light & 13 & 40.1 & 3.18 \\
\hline 5 & $12,038+12,039$ & A-12 & coarse-grained ophytic feldspar basalt & reflected light & 19 & 37.9 & 3.24 \\
\hline 6 & $14,073+14,076$ & A-14 & KREEP basalt (pyroxene) & transm. light & 229 & 27.8 & 3.88 \\
\hline 7 & 14,078 & A-14 & KREEP subophytic basalt & reflected light & 27 & 25.3 & 3.89 \\
\hline 8 & 15,016 & A-15 & medium-grained vesicular olivine-normative basalt & transm. light & 56 & 38.3 & 3.33 \\
\hline 9 & 15,603 & A-15 & olivine-normative basalt & transm. light & 84 & 41.4 & 3.27 \\
\hline 10 & 15,674 & A-15 & fine-grained olivine-normative basalt & transm. light & 48 & 36 & 3.37 \\
\hline 11 & 70,215 & A-17 & fine-grained mare basalt & reflected light & 22 & 28.3 & 3.7 \\
\hline 12 & $70,215-1$ & A-17 & fine-grained mare basalt & reflected light & 46 & 29.5 & 3.7 \\
\hline 13 & 75,055 & A-17 & coarse-grained and fine-grained ilmenitic basalt & reflected light & 5 & 30 & 3.83 \\
\hline 14 & 78,585 & A-17 & vitrophyric basalt & reflected light & 98 & 29.6 & 3.72 \\
\hline
\end{tabular}

Notes: The first column indicates the number of the lunar basalt samples; Apollo flight designates the Apollo flight number (the landing site and sampling); "reflected" refers to the photos of the thin sections in reflected light, "light" refers to the thin section in the transmitted light; $N$ is the number of measured grains of "iron"; $D$ is the average size of the grains of "iron" in $\mu \mathrm{m}$; the last columns designate the radiologically determined age of the basaltic samples (in billions of years ago). 


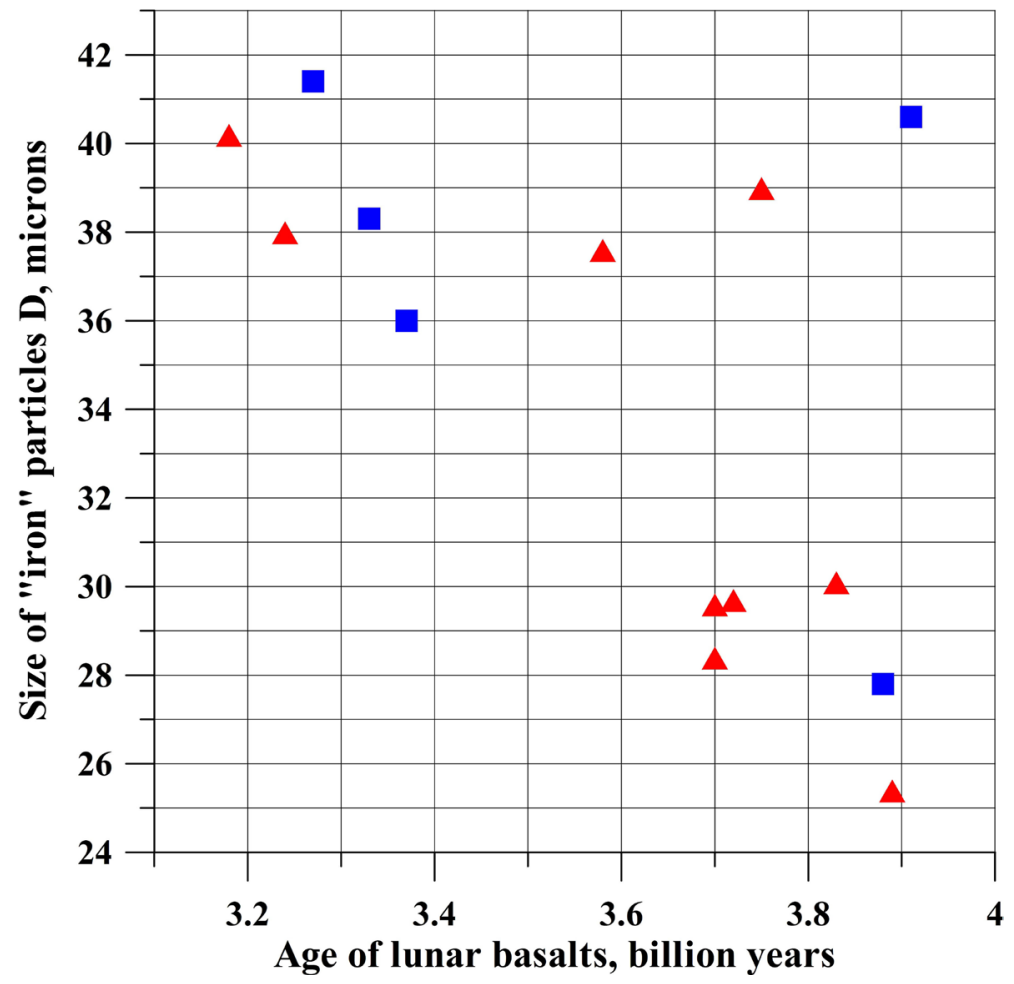

Figure 2. Dependence of the average grain size $D$ of "iron" in lunar basalts on the age of basalts. Red triangles designate samples determined by reflected light, and blue squares by the transmitted light.

alternatively its mass decreased, or that both these factors changed. Nevertheless, we cannot exclude the least trivial and most incredible explanation of a time variation in the gravitational constant (as we know, 80 years ago P. Dirac noted the possible changes of gravitational constant in time [7]; a situation with the gravitational constant is unclear till present [8]).

Interestingly that some researchers (for instance, [9]) indicate that the offset of the center of mass of the Moon from its center of figure toward the Earth during the considered geological time (3.8 - 3.0 eons: mare basalts [6]). These scientists have associated this phenomenon by the asymmetrical thinning of the lunar crust.

After the first rush of excitement, we decided to take another, calmer look at Figure 2 and Table 1 to analyze the global nature of the dependence of the grain size of the "iron" on the age of the lunar basalts. For example, in basalts of similar ages, there are specimens with similar $D$ from different Apollo missions; i.e., from different regions of the Moon (samples 10,003, 12,022, 15,603 are dated to 3 - $3.2 \mathrm{Ba}$ with a $D$ of $40-41 \mu \mathrm{m}$ whereas samples $14,073,14,078,75,055$ are dated roughly to $3.9 \mathrm{Ba}$ with a $D$ of $27-30 \mu \mathrm{m}$ ).

The variety of petrographic types of lunar basalts (Table 1) does not affect the relationship of the average grain size of these "iron" samples to the age of the basalts. For example, the predominance of ilmenite, which is lighter than iron, should lead to a coarsening of the average grain size, but among the ilmenite ba- 
salts there are samples with high $(D \approx 40 \mu \mathrm{m}$, samples $10,003,10,017)$ and low ( $D=30 \mu \mathrm{m}$, sample 75055) size particles. This is also true for non-ilmenite samples since both high average sizes $(D \approx 40 \mu \mathrm{m})$ were found for samples 12,038 and 15,603 , and low sizes $(D \approx 27 \mu \mathrm{m})$ for samples 14,073 and 14,078 .

Overall, the minimum average particle size of lunar iron grains was $27 \mu \mathrm{m}$, which only differs from the average size of the grains of terrestrial iron by $\sim 21$ $\mu \mathrm{m}$, and even the maximum average size of the lunar iron particles at $\sim 40 \mu \mathrm{m}$ is only twice as large as the average particle size of terrestrial iron $(21 \mu \mathrm{m})$, whereas the modern terrestrial gravity field is six times larger than the Moon's gravity field today. This may indicate a major influence of the Moon's radius to the growth $D$.

\section{Conclusion}

The consistency of the average size of "iron" particles in terrestrial basalts and in sediments, which is expressed in a comparable average particle size of $\sim 21 \mu \mathrm{m}$, and the same mode of $10-20 \mu \mathrm{m}$ is assumed to be associated with the constant effect of gravity during the precipitation of "iron" particles in sediments and basaltic lava. To verify this association, an object with a completely different $g$, such as the basalts occurring on the Moon whose $g$ is 6 times smaller than on Earth was needed. Surprisingly we discovered a significant dependence of the average size of lunar "iron" grains on the age of the lunar basalts, and consequently on $g$. from 3.9 to $3.4 \mathrm{Ba}$, the average grain size of "iron" increased by 1.5 fold, and $g$ diminished, suggesting that the size of the Moon grew, its mass decreased, or both these factors. This dependence emerged as global in nature at least for the Moon. Considering the essential changes of $g$ over time, the $g$ of synchronous basalts needs to be compared, but since there are no young basalts on the Moon, terrestrial basalts of a similar age need to be studied; i.e., samples dated to $3-4 \mathrm{Ba}$. If such a significant change in gravity acceleration is specific to the Moon, and perhaps to other stellar bodies such as the Earth, or even the entire Solar system. This can be measured fairly easily on the Earth: we need a series of samples of terrestrial basalts with a known radiological age down to $3-4 \mathrm{Ba}$, better prepared sections, and observations using a microprobe to obtain an unambiguous selection of grains of metallic "iron". We cannot exclude also the most incredible explanation of changing the gravitational constant with time.

\section{Acknowledgements}

We thank N. A. Krivolutskaya for her assistance in researching the data on lunar basalts.

\section{Conflicts of Interest}

The authors declare no conflicts of interest regarding the publication of this paper. 


\section{References}

[1] Pilchin, A.N. and Eppelbaum, L.V. (2006) Iron and Its Unique Role in the Earth Evolution. Mexico Geoph. Society, Mexico.

[2] Pechersky, D.M. (2012) Native Iron and Other Magnetic Minerals in Sediments: Thermomagnetic Signs of Cosmic Origin. Palmarium Academic Publishing, Saarbrücken.

[3] Pechersky, D.M. (2015) Distribution of Native Iron Particles and Fe-Ni Alloys on Planets. Palmarium Academic Publishing, Saarbrücken.

[4] Kramer, F.E., Twedell, D.B. and Walton, W.J.A. (1977) Apollo 11 Lunar Sample Information Catalogue (Revised). Curator's Office, JSC 12522, Houston.

[5] Halliday, A.N. (2012) The Origin of the Moon. Science, 338, 1040-1041. https://doi.org/10.1126/science.1229954

[6] Schmitt, H.H. (1991) Evolution of the Moon: Apollo Model. American Mineralogist, 76, 773-784.

[7] Dirac, P.A.M. (1938) A New Basis for Cosmology. Proceedings of the Royal Society $A, 165,199-208$. https://doi.org/10.1098/rspa.1938.0053

[8] Anderson, J.D., Schubert, G., Trimble, V. and Feldman, M.R. (2015) Measurements of Newton's Gravitational Constant G and the Length of Day. Europhysics Letters, 110, Article ID: 10002. https://doi.org/10.1209/0295-5075/110/10002

[9] Kaula, W.M., Schubert, B., Lingenfelter, R.E., Sjogren, W.L. and Wollenhaupt, W.R. (1974) Apollo Laser Altimetry and Inferences as to Lunar Structure. Proceedings of the 5 th Lunar Science Conference, 3, 3049-3058. 